\title{
Einstein-Rosen Bridge (ER), Einstein-Podolsky-Rosen Experiment (EPR) and Zero Measure Rindler-KAM Cantorian Spacetime Geometry (ZMG) Are Conceptually Equivalent
}

\author{
Mohamed S. El Naschie \\ Department of Physics University of Alexandria, Alexandria, Egypt \\ Email: Chaossf@aol.com
}

Received 22 December 2015; accepted 5 January 2016; published 8 January 2016

Copyright (C) 2016 by author and Scientific Research Publishing Inc.

This work is licensed under the Creative Commons Attribution International License (CC BY).

http://creativecommons.org/licenses/by/4.0/

(c) (i)

\section{Abstract}

By viewing spacetime as a transfinite Turing computer, the present work is aimed at a generalization and geometrical-topological reinterpretation of a relatively old conjecture that the wormholes of general relativity are behind the physics and mathematics of quantum entanglement theory. To do this we base ourselves on the comprehensive set theoretical and topological machinery of the Cantorian-fractal E-infinity spacetime theory. Going all the way in this direction we even go beyond a quantum gravity theory to a precise set theoretical understanding of what a quantum particle, a quantum wave and quantum spacetime are. As a consequence of all these results and insights we can reason that the local Casimir pressure is the difference between the zero set quantum particle topological pressure and the empty set quantum wave topological pressure which acts as a wormhole "connecting" two different quantum particles with varying degrees of entanglement corresponding to varying degrees of emptiness of the empty set (wormhole). Our final result generalizes the recent conceptual equation of Susskind and Maldacena ER $=$ EPR to become

$$
\text { ZMG = ER = EPR }
$$

where ZMG stands for zero measure Rindler-KAM geometry (of spacetime). These results were only possible because of the ultimate simplicity of our exact model based on Mauldin-Williams random Cantor sets and the corresponding exact Hardy's quantum entanglement probability $P(H)$ $=\phi^{5}$ where $\phi$ is the Hausdorff dimension of the topologically zero dimensional random Cantor thin set, i.e. a zero measure set and $\phi=(\sqrt{5}-1) / 2$. On the other hand the positive measure spatial 
separation between the zero sets is a fat Cantor empty set possessing a Hausdorff dimension equal $\phi^{2}$ while its Menger-Urysohn topological dimension is a negative value equal minus one. This is the mathematical quintessence of a wormhole paralleling multiple connectivity in classical topology. It is both physically there because of the positive measure and not there because of the negative topological dimension.

\section{Keywords}

Zero Measure Thin Cantor Set, Fat Cantor Set, Cantorian Fractal KAM Spacetime, Quantum Gravity, Casimir Pressure, E-Infinity Theory

\section{Introduction}

The present paper had its roots around six years ago in our work on the geometrical-topological interpretation of Hardy's famous quantum entanglement and transfinite golden mean Turing computers [1] as well as its connection to a fractal version of Rindler spacetime combined with KAM theorem of nonlinear dynamics [2]. This work led to an exact determination of the ordinary and the dark energy density of the cosmos [2]-[6]. Shortly after that we started exploring the possibility that zero measure geometry could be interpreted as a wormhole [2]. This work was carried out admittedly in total ignorance of classical contributions on the subject [7]-[31]. In fact it was only the remarkable papers of Profs. L. Susskind and J. Maldacena which made us aware that we are not working in a vacuum [27].

One idea led to another and the logic of the situation set the course at attempting not only to validate the remarkable suggestions of Susskind and Maldacena but also to reduce their results to a natural consequence of a Cantorian Rindler-KAM zero measure and empty set geometry. Actually this is a grossly simplified label because the required E-infinity-Rindler-KAM spacetime is a multi fractal containing sets with positive and zero measure, $\mathrm{u}$ thin and fat fractals as well as positive and negative topological Menger-Urysohn dimensions as explained in the extensive literature on E-infinity theory and its application [32]-[118], particularly to spacetime Casimir-dark energy reactors [63]-[71] and transfinite Turing computers [120] [121].

\section{Transfinite Set Theoretical Conception of Quantum Spacetime}

Our general theory of quantum spacetime follows in a rigorous way from the von Neumann-Connes by now famous dimensional group function [119]

$$
D=a+b \phi
$$

where $a, b, \in Z$ and $\phi=(\sqrt{5}-1) / 2$. The above may be written in a simple compact notation known as the bijection formula which states that [1]-[5]

$$
d_{c}^{(n)}=(1 / \phi)^{n-1}
$$

where $d_{c}=D_{H}$ is the Hausdorff dimension corresponding to the Menger-Urysohn topological dimension n. For instance if we take $n=O$ which is a zero set modelling the quantum (pre)particle, we see that [1]-[5] [119]

$$
\begin{aligned}
& d^{(o)}=(1 / \phi)^{-1} \\
& =\phi
\end{aligned}
$$

On the other hand, for $n=-1$ which is the classical empty set and in our theory models the (pre)quantum wave, we see that

$$
\begin{aligned}
& d_{c}^{(-1)}=(1 / \phi)^{-1-1} \\
& =\phi^{2}
\end{aligned}
$$

Two further particular dimensions are of relevance to the present work, namely $n=-\infty$ for which we have clearly 


$$
d_{c}^{(-\infty)}=\phi^{\infty}
$$

$=$ zero

and

$$
\begin{aligned}
& d_{c}^{(4)}=(1 / \phi)^{4-1} \\
& =(1 / \phi)^{3} \\
& =4+\phi^{3} \\
& =4+\frac{1}{4+\frac{1}{4+\ldots}} \\
& =4.2360679
\end{aligned}
$$

Adding to the above the realization gained from the elementary theory of co-bordism [74] we see that the surface of the zero set $\mathrm{D} \equiv(O, \phi)$ is the empty set $\mathrm{D} \equiv\left(-1, \phi^{2}\right)$ [69] [70] [74] [86]. In turn the surface of the empty set is the emptier set $\mathrm{D} \equiv\left(-2, \phi^{3}\right)$. On the other hand the average set of all sets is given by $\phi^{3}$ so that it must be spacetime itself. To see this subtle point we look at the inversion of $\phi^{3}$ which is [32]

$$
1 / \phi^{3}=\mathbf{4}+\phi^{3} \text {. }
$$

In other words it gives us the average spacetime dimension [32]

$$
\begin{aligned}
& \langle d c\rangle=\frac{\sum_{o}^{\infty} n^{2} \phi^{n}}{\sum_{o}^{\infty} n \phi^{n}} \\
& =\sum_{n=0}^{\infty} n \phi^{n} \\
& =4+\phi^{3}
\end{aligned}
$$

That way we can construct a very simple and beautiful mental picture of our universe [73]-[76]. First we see a quantum pre-particle resembling a micro black hole fixed by $D=(O, \phi)$ which is the zero set. The surface surrounding the zero set is the empty set (pre)quantum wave $D=\left(-1, \phi^{2}\right)$. Then we see that the surface of the wave is a multi fractal with average bi dimension $\langle D\rangle=\left\langle\left(-2, \phi^{3}\right)\right\rangle$ and this is nothing else but our quantum spacetime itself where the particle resides "inside" a wave floating in spacetime, which is its fractal surface. Now we ask ourselves the natural questions about the surface of spacetime. This is clearly $\langle D\rangle=\langle(-\infty, O)\rangle$ which means nothingness, i.e. where there are no answers because there are no questions [74]. Next we contemplate the place of a wormhole in the above using the terminology of set theory and measure theory.

\section{What Is a Wormhole from the View Point of Measure Theory?}

It is almost a trivial conclusion that a wormhole [7]-[31] is the quintessential empty set with varying degrees of emptiness corresponding to varying degrees of entanglement [1] [37] [45]. Our spacetime consists exclusively of zero sets quantum particles and empty sets quantum waves. Consequently the infinitely many empty sets with positive measure are of negative dimensions and thus somewhat esoteric although they have a positive measure but in negative dimensions as a wormhole connecting two zero sets quantum particles. One could envisage the situation by looking at a single Cantor set. Imagine removing the middle third "randomly" as we do in the conventional construction of a one dimensional random Cantor set. Now the Hausdorff dimension of the "gaps" is $1-\phi=\phi^{2}$. That means it is an empty set, in fact in this case, total nothingness [74]. Now at end "points" we have a Hausdorff dimension of a zero set particle, namely $\phi$. Thus we have a topological pressure gradient acting inside the "gap", i.e. the empty set which we can now call the wormhole and the gradient is obviously equal $\phi-\phi^{2}$ which is $\phi^{3}$. Again $\phi^{3}$ is not only the universal fluctuation of spacetime [32] [74] but also equal to the local Casimir effect which we should call henceforth, the Casimir pressure. When all is said and done, mathe- 
matically a wormhole is best modelled by a totally empty set given by the bi-dimension $(-\infty, O)$ or at most an empty set $\left(-1, \phi^{2}\right)$ and with all fat empty Cantor sets in between these two limits [74]. The important notions of fat Cantor sets and wild topology is explained in detail in Ref [55].

\section{From Local Casimir Pressure to Globally Concentrated Dark Energy}

The Casimir topological pressure $\phi^{3}$ is clearly a spacetime latent pressure which is the difference between the average Hausdorff dimension of spacetime and the Menger-Urysohn dimension of the same. That means it is $\left(4+\phi^{3}\right)-4=\phi^{3}$. Locally it is explained as the difference between the zero set $\phi$ and the empty set $\phi^{3}$ which means again $\phi-\phi^{2}=\phi^{3}$. However, at the "end" of the universe we have a one sided Möbius like boundary separating $\phi^{3}$ from nothingness. Taking the measure concentration theorem of Dvoretzky into account [100] [101] we have one sided topological pressure causing expansion. It then turned out that almost $96 \%$ of energy is at the boundary of the holographic boundary and is given exactly by the dark energy density $E(D)=5 \phi^{2} / 2\left(m c^{2}\right)$ while the rest is inside the "universe" and is given by the ordinary energy density $E(O)=\left(\phi^{5} / 2\right)\left(m c^{2}\right)$ as discussed in great detail in [10] [88] and the references therein.

\section{The Dimensionality of Spacetime from a Fractal Strings Interpretation of E-Infinity Theory}

As mentioned before, there are two basic sets in E-infinity theory, the zero set $D \equiv(O, \phi)$ and the empty set $D_{-1} \equiv\left(-1, \phi^{2}\right)$. By the usual inversion one moves to positive "observable" space dimensions, namely to a fractal string given Hausdorffly by $d_{c}^{(2)}=1 / \phi=1+\phi$ and $d_{c}^{(3)}=1 / \phi^{2}=2+\phi$. In other words the zero pre-quantum particle transmutes to a fractal string with a dimension $1+\phi=1.618033989 \ldots$ and the empty pre-quantum wave transmutes to a fractal world sheet $2+\phi=2.618033989 . .$. . Our E-infinity spacetime expectation dimension, i.e. average multi fractal dimension is then found either as the union or the intersection of the fractal string "particle" with the fractal world sheet "wave” [32] [74]. That means

$$
\begin{aligned}
& D=(1+\phi) \oplus(2+\phi) \\
& =4+\phi^{3}
\end{aligned}
$$

or

$$
\begin{aligned}
& D=(1+\phi) \otimes(2+\phi) \\
& =4+\phi^{3}
\end{aligned}
$$

This is the remarkable indistinguishability condition of E-infinity spacetime [32] which stresses the intrinsic fuzziness of this space where intersection and union give exactly the same result and leads to the famous outcome of all "which way" quantum experiment. Nature is not paradoxical but only irreducibly fuzzy on the fundamental quantum scale. It is both chaotic and deterministic. In the terminology of G. 'tHooft, we are simply dealing with a deterministic quantum mechanics and the conceptual equality [16]

$$
\mathrm{EP}=\mathrm{EPR}=\mathrm{ZMG}
$$

is just another manifestation of this geometrical-topological fractal-Cantorian fuzziness.

\section{Conclusion}

We have known since a long time that orthodox quantum mechanics has no place for our human intuitive need for a concept related to spatial separation. However, even general relativity evades this concept and leaves the possibility open by contemplating a multiply connected topology and consequently the possible existence of wormholes [7]-[37]. It did not take long before many researchers started to suspect that quantum entanglement of wormholes may be two sides of the same coin. String theory offered a theoretical possibility which could not be overlooked by pioneers like Susskind and Maldacena. In fact speculation on such a connection leading to a theory of quantum gravity was discussed in the excellent text book of Prof. P. Holland [114] on the quantum theory of motion which is an account of the de Broglie-Bohm causal interpretation of quantum mechanics and therefore in many respects, related to the deterministic quantum mechanics of Nobel Laureate G. 'tHooft [115] and the fractal-Cantorian indeterministic classical mechanics proposed by the present author [116] as well as G. 
Ord and L. Nottale [32]. However, in the Cantorian version of this theory exposed here we go one step further. We are not just satisfied by showing that quantum entanglement is a form of a wormhole or vice versa. We want to show that both quantum entanglement and wormholes are manifestations of something fundamental to mathematical logic as expressed in set theory and one of its main off springs, namely measure theory and the Menger-Urysohn dimensional theory [74]. It is time and time again the same problem which only a few gifted mathematicians of the calibre of von Neumann and Alain Connes [119] noticed and could deal with as they are interested in physics but are also well aware of the definite difference between zero, empty and nothingness [74]. It is as simple and as difficult as that and nothing more but also nothing less than taking pure mathematics very seriously when working on foundational deep problems in physics. Let us conclude this paper by stressing again that mathematically a wormhole is an empty set with varying degrees of emptiness. It is not zero measure because it is a fat fractal [55]. However, it provides no classical spatial separation because it is a positive measure of an empty set converging to nothing and living in a somewhat difficult to classically imagine negative topological dimension. It is really there and not there at the same time. It is like life itself: one moment we are there and in a split of a second we are not there although the universe as a whole still goes on unperturbed by us ceasing to exist.

\section{Acknowledgements}

Without the work of Professors Susskind, Maldacena, 'tHooft and the excellent book of Prof. Holland this paper could not have seen the light. To them and all the authors referred to in this paper I am truly indebted.

\section{References}

[1] Mohamed S. El Naschie: Quantum Entanglement as a Consequence of a Cantorian Micro Spacetime Geometry. Journal of Quantum Information Science, 1(2), 2011, pp. 50-53.

[2] Mohamed S. El Naschie: A Rindler-KAM Spacetime Geometry and Scaling the Planck Scale Solves Quantum Relativity and Explains Dark Energy. International Journal of Astronomy and Astrophysics, 3(4), 2013, pp. 483-493.

[3] Mohamed S. El Naschie: What Is the Missing Dark Energy in a Nutshell and the Hawking-Hartle Quantum Wave Collapse. International Journal of Astronomy and Astrophysics, 3(3), 2013.

[4] L. Marek-Crnjac, M. S. El Naschie: Quantum Gravity and Dark Energy Using Fractal Planck Scaling. Journal of Modern Physics, 4(11A), 2013, pp. 31-38.

[5] M. S. El Naschie and Ji-Huan He: Quantum Gravity and Dark Energy via a New Planck Scale. Journal of Fractal Spacetime \& Noncommutative Geometry in Quantum \& High Energy Physics, 3(2), 2013, pp. 106-119.

[6] Mohamed S. El Naschie: The Missing Dark Energy of the Cosmos from Light Cone Topological Velocity and Scaling of the Planck Scale. Open Journal of Microphysics, 3(3), 2013.

[7] M. Van Raamsdonk: Building up Space-Time with Quantum Entanglement. International Journal of Modern Physics D, 19, 2010, pp. 2429.

[8] T. C. Ralph, G. J. Milburn and T. Downes: Quantum Connectivity of Space-Time and Gravitationally Induced Decorrelation of Entanglement. Physical Review A., 79(2), 2009, pp. 022121.

[9] M. Requardt: Wormhole Spaces: the Common Cause for the Black Hole Entropy-Area Law, the Holographic Principle and Quantum Entanglement. arXiv preprint arXiv:0910.4017, 2009

[10] K. Jensen and A. Karch: Holographic Dual of an Einstein-Podolsky-Rosen Pair Has a Wormhole. Physical Review Letters, 111, 2013, pp. 211602.

[11] T. Takayanagi: Entanglement Entropy from a Holographic Viewpoint. Classical and Quantum Gravity, 29(15), 2012.

[12] D. Marolf and J. Polchinski: Gauge-Gravity Duality and the Black Hole interior. Physical Review Letters, 111, 2013, pp. 171301.

[13] M. Chernicoff, A. Güijosa and J. F. Pedraza: Holographic EPR Pairs, Wormholes and Radiation. Journal of High Energy Physics, 10, 2013, p. 211.

[14] B. Czech, J. L. Karczmarek, F. Nogueira and M. Van Raamsdonk: Rindler Quantum Gravity. Classical and Quantum Gravity, 29(23), 2012.

[15] L. J. Garay: Quantum Evolution in Space-Time Foam. International Journal of Modern Physics A, 14(26), 1999.

[16] H. Gharibyan, R. F. Penna: Are Entangled Particles Connected by Wormholes? Evidence for the ER = EPR Conjecture from Entropy Inequalities. Physical Review D, 89, 2014, pp. 066001. 
[17] B. Swingle: Constructing Holographic Spacetimes Using Entanglement Renormalization. arXiv Preprint arXiv: 1209.3304, 2012.

[18] V. Balasubramanian and P. Hayden: Multiboundary Wormholes and Holographic Entanglement. Classical and Quantum Gravity, 31(18), 2014.

[19] S. Coleman: Black Holes as Red Herrings: Topological Fluctuations and the Loss of Quantum Coherence. Nuclear Physics B, 4(3), 1988, pp. 867-882.

[20] E. Joos, H. D. Zeh, C. Kiefer, D. J. W. Giulini, J. Kupsch: Decoherence and the Appearance of a Classical World in Quantum Theory. Springer, Heidelberg, Germany, 2003.

[21] P. Pizzi: Spacetime at the Planck Scale: The Quantum Computer View. The Foundations of Quantum Mechanics: Historical, 2004.

[22] M. B. Cantcheff: Emergent Spacetime, and a Model for Unitary Gravitational Collapse in AdS. arXiv Preprint arXiv:1110.0867, 2011.

[23] B. Swingle: Entanglement Renormalization and Holography. Physical Review D, 86, 2012, pp. 065007.

[24] M. Requardt: Wormhole Spaces, Connes' "Points, Speaking to Each Other”, and the Translocal Structure of Quantum Theory. arXiv preprint hep-th/0205168, 2002

[25] J. C. Baez and J. Vicary: Wormholes and entanglement. Classical and Quantum Gravity, 31(21), 2014.

[26] S. Seki and S.J. Sin: EPR= ER, scattering amplitude and entanglement entropy change. Physics Letters B, 735, 2014, pp. 272-276.

[27] L. Susskind: Entanglement Is Not Enough. arXiv preprint arXiv:1411.0690, 2014

[28] E. S. Santini: Might EPR Particles Communicate through a Wormhole? EPL (Europhysics Letters), 78(3), 2007.

[29] J. Sonner: Holographic Schwinger Effect and the Geometry of Entanglement. Physical Review Letters, 111, 2013, pp. 211603.

[30] J. Aron: Wormhole Entanglement Solves Black Hole Paradox. New Scientist, 218(2922), 2013, pp. 9.

[31] S. Durand: An Amusing Analogy: Modelling Quantum-Type Behaviours with Wormhole-Based Time Travel. Journal of Optics B: Quantum and Semiclassical Optics, 4(4), 2002.

[32] M. S. El Naschie: A Review of E-Infinity and the Mass Spectrum of High Energy Particle Physics. Chaos, Solitons \& Fractals, 19(1), 2004, pp. 209-236.

[33] M. S. El Naschie: On the Uncertainty of Cantorian Geometry and the Two-Slit Experiment. Chaos, Solitons \& Fractals, 9(3), 1998, pp. 517-529.

[34] M. S. El Naschie: Superstrings, Knots and Noncommutative Geometry in E-Infinity Space. International Journal of Theoretical Physics, 37(12), 1998, pp. 2935-2951.

[35] Mohamed S. El Naschie: Quantum Mechanics and the Possibility of a Cantorial Spacetime. Chaos, Solitons \& Fractals, 1(5), 1991, pp. 485-487.

[36] M. S. El Naschie: On a Fuzzy Kähler-Like Manifold which Is Consistent with the Two Slit Experiment. International Journal of Nonlinear Sciences and Numerical Simulation, 6(2), 2005, pp. 95-98.

[37] M. S. El Naschie: The Theory of Cantorian Spacetime and High Energy Particle Physics (An Informal Review). Chaos, Solitons \& Fractals, 41(5), 2009, pp. 2635-2646.

[38] M. S. El Naschie, O. E. Rössler and I. Prigogine: Quantum Mechanics, Diffusion and Chaotic Fractals. Pergamon Press/Elsevier, Oxford ISBN: 0080420273, 1995.

[39] M. S. El Naschie: Stress, Stability and Chaos in Structural Engineering. McGraw Hill, London, 1990.

[40] M. S. El Naschie: Non-Euclidean Spacetime Structure and the Two-Slit Experiment. Chaos, Solitons \& Fractals, 26, 2005, pp. 1-6.

[41] M. S. El Naschie: On the Unification of Heterotic Strings, M Theory and E ( $\infty$ ) Theory. Chaos, Solitons \& Fractals, 11(14), 2000, pp. 2397-2408.

[42] M. S. El Naschie: Elementary Prerequisites for E-Infinity (Recommended Background Readings in Nonlinear Dynamics, Geometry and Topology). Chaos, Solitons \& Fractals, 30, 2006, pp. 579-605.

[43] M. S. El Naschie: The Concepts of E-Infinity: An Elementary Introduction to the Cantorian-Fractal Theory of Quantum Physics. Chaos, Solitons \& Fractals, 22, 2004, pp. 495-511.

[44] M. S. El Naschie: On a Class of General Theories for Higher Energy Particle Physics. Chaos, Solitons \& Fractals, 14, 2002, pp. 649-668.

[45] M. S. El Naschie: Towards a General Transfinite Set Theory for Quantum Mechanics. Fractal Spacetime and Noncommutative Geometry in Quantum and High Energy Physics, 2(2), 2012, pp. 135-142. 
[46] M. S. El Naschie: Symmetry Group Prerequisite for E-Infinity in high Energy Physics. Chaos, Solitons \& Fractals, 35(1), 2008, pp. 202-211.

[47] M. S. El Naschie: The Feynman Path Integral and E-Infinity from Two-Slit Gedanken Experiment. Int. J. Nonlinear Science \& Numerical Systems, 6(4), 2005, pp. 335-342.

[48] M. S. El Naschie: A Guide to the Mathematics of E-Infinity Cantorian Spacetime Theory. Chaos, Solitons \& Fractals, 25, 2005, pp. 935-964.

[49] M. S. El Naschie: Quantum Gravity, Clifford Algebras, Fuzzy Set Theory and the Fundamental Constants of Nature. Chaos, Solitons \& Fractals, 20(3), 2004, pp. 437-450.

[50] M. S. El Naschie: Elementary Number Theory in Superstring Loop Quantum Mechanics, Twistors and E-Infinity High Energy Physics. Chaos, Solitons \& Fractals, 27(2), 2006, pp. 297-330.

[51] M. S. El Naschie: Time Symmetry Breaking, Duality and Cantorian Spacetime. Chaos, Solitons \& Fractals, 7(4), 1996, 499-518.

[52] M. S. El Naschie: Exceptional Lie Groups Hierarchy and the Structure of the Micro Universe. International Journal of Nonlinear Science \& Numerical Simulation, 8(3), 2007, pp. 445-450.

[53] M. S. El Naschie: Topics in the Mathematical Physics of E-Infinity Theory. Chaos, Solitons \& Fractals, 30(3), 2006, pp. 656-663.

[54] M. S. El Naschie: Is Quantum Space a Random Cantor Set with a Golden Mean Dimension at the Core? Chaos, Solitons \& Fractals, 4(2), 1994, pp. 177-179.

[55] M. S. El Naschie: Wild Topology, Hyperbolic Geometry and Fusion Algebra of High Energy Particle Physics. Chaos, Solitons \& Fractals, 13(9), 2009, pp. 1935-1945.

[56] M. S. El Naschie: From Experimental Quantum Optics to Quantum Gravity via a Fuzzy Kähler Manifold. Chaos, Solitons \& Fractals, 25(5), 2005, pp. 969-977.

[57] M. S. El Naschie: On the Unification of the Fundamental Forces and Complex Time in E-Infinity Space. Chaos, Solitons \& Fractals, 11, 2000, pp. 1149-1162.

[58] M. S. El Naschie: Quantum Gravity from Descriptive Set Theory. Chaos, Solitons \& Fractals, 19(5), 2004, pp. 1339-1344.

[59] M. S. El Naschie: A Note on Quantum Mechanics, Diffusional Interference and Information. Chaos, Solitons \& Fractals, 5(5), 1995, pp. 881-884.

[60] M. S. El Naschie: Introduction to Chaos, Information and Diffusion in Quantum Physics. Chaos, Solitons \& Fractals, 7(5), 1996, pp. vii-x.

[61] M. S. El Naschie: On Dimensions of Cantor Set Related Systems. Chaos, Solitons \& Fractals, 3(6), 1993, pp. 675-685.

[62] Leila Marek-Crnjac: On El Naschie’s Fractal-Cantorian Space-Time and Dark Energy-A Tutorial Review. Natural Science, 7(13), 2015.

[63] M. S. El Naschie: Casimir-Dark Energy Nano Reactor Design Proposal Based on Fractals. International Journal of Innovation is Science and Mathematics, 3(4), 2015, pp. 2347-9051.

[64] M. S. El Naschie: The Counterintuitive Increase of Information Due to Extra Spacetime Dimensions of a Black Hole and Dvoretzky’s Theorem. Natural Science, 7(10), 2015.

[65] Mohamed S. El Naschie: Application of Dvoretzky’s Theorem of Measure Concentration in Physics and Cosmology. Open Journal of Microphysics, 5, 2015, pp. 11-15.

[66] Mohamed S. El Naschie: A Resolution of the Black Hole Information Paradox via Transfinite Set Theory. World Journal of Condensed Matter Physics, 5, 2015, pp. 249-260.

[67] Mohamed S. El Naschie: If Quantum "Wave” of the Universe then Quantum "Particle” of the Universe: A Resolution of the Dark Energy Question and the Black Hole Information Paradox. International Journal of Astronomy \& Astrophysics, 5, 2015, pp. 243-247.

[68] Mohamed S. El Naschie: Quantum Fractals and the Casimir-Dark Energy Duality-The Road to a Clean Quantum Energy Nano Reactor. Journal of Modern Physics, 6, 2015, pp. 1321-1333.

[69] Mohamed S. El Naschie: From Fusion Algebra to Cold Fusion or from Pure Reason to Pragmatism. Open Journal of Philosophy, 5(6), 2015.

[70] M. S. El Naschie: The Casimir Effect as a Pure Topological Phenomenon and the Possibility of a Casimir Nano Reactor-Design. American Journal of Nano Research and Application, 3(3), 2015, pp. 33-40.

[71] M. S. El Naschie: Cosserat-Cartan and de Sitter-Witten Spacetime Setting for Dark Energy. Quantum Matter, 5(1), 2016, pp. 1-4. 
[72] Mohamed S. El Naschie: Hubble Scale Dark Energy Meets Nano Scale Casimir Energy and the Rational of Their T-Duality and Mirror Symmetry Equivalence. World Journal of Nano Science and Engineering, 5, 2015, pp. 57-67.

[73] Mae-wan Ho, Mohamed El Naschie and Giueseppe Vitiello: Is Spacetime Fractal and Quantum Coherent in the Golden mean. Global Journal of Science Frontier Research-A: Physics and Space Science, 15(1), 2015, pp. 61-80.

[74] Mohamed S. El Naschie: An Exact Mathematical Picture of Quantum Spacetime. Advances in Pure Mathematics, 5, 2015, pp. 560-570.

[75] Mohamed S. El Naschie: On a Non-Perturbative Quantum Relativity Theory Leading to a Casimir-Dark Energy Nanotech Reactor Proposal. Open Journal of Applied Science, 5(7), 2015.

[76] M. S. El Naschie: The Cantorian Monadic Plasma behind the Zero Point Vacuum Spacetime Energy. American Journal of Nano Research and Application, 3(3), 2015, pp. 66-70.

[77] M. S. El Naschie: A Cold Fusion-Casimir Energy Nano Reactor Proposal. World Journal of Nano Science and Engineering, 5, 2015, pp. 49-56.

[78] M. S. El Naschie: A Casimir-Dark Energy Nano Reactor Design—Phase I. Natural Science, 7, 2015, pp. 287-298.

[79] M. S. El Naschie: Fuzzy Multi-Instanton Knots in the Fabric of Spacetime and Dirac's Vacuum Fluctuation. Chaos, Solitons \& Fractals, 38, 2008, pp. 1260-1268.

[80] M. S. El Naschie: Kerr Black Hole Geometry Leading to Dark Matter and Dark Energy via E-Infinity Theory and the Possibility of Nano Spacetime Singularity Reactor. Natural Science, 7(4), 2015, pp. 210-225.

[81] Jean-Paul Auffray: E-Infinity, the Zero Set, Absolute Space and the Photon Spin. Journal of Modern Physics, 6(5), 2015, pp. 536-545.

[82] M. S. El Naschie: A Fractal Rindler-Regge Triangulation in the hyperbolic Plane and Cosmic de Sitter Accelerated Expansion. Journal of Quantum Information Science, 5(1), 2015, pp. 24-31.

[83] M. S. El Naschie: Computing Dark Energy and Ordinary Energy of the Cosmos as a Double Eigenvalue Problem. Journal of Modern Physics, 6(4), 2015, pp. 348-395.

[84] M. S. El Naschie: The Casimir Topological Effect and a Proposal for a Casimir-Dark Energy Nano Reactor. World Journal of Nano Science and Engineering, 5(1), 2015, pp. 26-33.

[85] M. S. El Naschie: From Kantian-Reinen Vernunft to Real Dark Energy Density of the Cosmos via Measure Concentration of Convex Geometry in Quasi-Banach Spaces. Open Journal of Philosophy, 5(1), 2015, pp. 123-130.

[86] A. P. Balachandran, S. Kürkcüoglu, S. Vaidya: Lectures on Fuzzy and Fuzzy Susy Physics. World Scientific, Singapore, 2007.

[87] M. S. El Naschie: Dark Energy and Its Cosmic Density from Einstein’s Relativity and Gauge Fields Renormalization Leading to the Possibility of a New 'tHooft Quasi Particle. The Open Journal of Astronomy, 8, 2015, pp. 1-17.

[88] Mohamed S. El Naschie: The Measure Concentration of Convex Geometry in a Quasi Banach Spacetime behind the Supposedly Missing Dark Energy of the Cosmos. American Journal of Astronomy \& Astrophysics, 2(6), 2014, pp. 72-77.

[89] S. Tharmar: Fuzzy I Vf-sets and fuzzy I ^ f-sets. International Journal of Basic Sciences and Applied Computing, 1(2), 2014.

[90] Mohamed S. El Naschie: From $E=m c^{2}$ to $E=m c^{2} / 22-A$ Short Account of the Most Famous Equation in Physics and Its Hidden Quantum Entanglement Origin. Journal of Quantum Information Science, 4, 2014, pp. 284-291.

[91] Jean-Paul Auffray: On an Intriguing Invention Albert Einstein Made Which Has Gone Unnoticed Hitherto. Journal of Modern Physics, 6(11), 2015, pp. 1478-1491.

[92] Mohamed S. El Naschie: The Self Referential Pointless Universe Geometry as the Key to the Resolution of the Black Hole Information Paradox. International Journal of Innovation in Science and Mathematics, 3(5), 2015, pp. $254-265$.

[93] L. M. Wapner: The Pea and the Sun. A. K. Peters Ltd., Wellesley, MA, USA, 2005.

[94] Mohamed S. El Naschie: A Complementarity Resolution of the Black Hole Information Paradox. American Journal of astronomy and Astrophysics, 3(5), 2015, pp. 77-86.

[95] M. S. El Naschie: The Cantorian Monadic Plasma behind the Zero Point Vacuum Spacetime Energy. American Journal of Nano Research \& Application, 3, 2015, pp. 66-70.

[96] A. J. Babchin and M. S. El Naschie: On the Real Einstein Beauty E $=$ kmc $^{2}$. World Journal of Condensed Matter Physics, 6(1), 2016.

[97] Mohamed S. El Naschie: Asymptotically Safe Pure Gravity as the Source of Dark Energy of the Vacuum. Int. Journal Astrophysics \& Space Science, 2(1), 2014, pp. 12-15.

[98] Mohamed S. El Naschie: Hardy’s Entanglement as the Ultimate Explanation for the Observed Cosmic Dark Energy and Accelerated Expansion. International Journal High Energy Physics, 1(2), 2014, pp. 13-17. 
[99] Mohamed S. El Naschie: Compactified Dimensions as Produced by Quantum Entanglement, the Four Dimensionality of Einstein's Smooth Spacetime and 'tHooft's 4- $\varepsilon$ Fractal Spacetime. American Journal of Astronomy \& Astrophysics, 2(3), 2014, pp. 34-37.

[100] Mohamed S. El Naschie: Banach Spacetime-like Dvoretzky Volume Concentration as Cosmic Holographic Dark Energy. International Journal of High Energy Physics, 2(1), 2015, pp. 13-21.

[101] Alexey Stakhov: The Mathematics of Harmony. World Scientific, Singapore, 2009.

[102] Mohamed S. El Naschie: The Hidden Quantum Entanglement Roots of $\mathrm{E}=\mathrm{mc}^{2}$ and Its Genesis to $\mathrm{E}=\mathrm{mc}^{2} / 22 \mathrm{plus}^{2}$ $\mathrm{mc}^{2}(21 / 22)$ Confirming Einstein's Mass-Energy Formula. American Journal of Electromagnetics and Applications, 2(5), 2014, pp. 39-44.

[103] Mohamed S. El Naschie: On a Casimir-Dark Energy Nano Reactor. American Journal of Nano Research and Application, 3(2), 2015, pp. 12-16.

[104] Mohamed S. El Naschie: Three Quantum Particles Hardy Entanglement from the Topology of Cantorian-Fractal Spacetime and the Casimir Effect as Dark Energy_A Great Opportunity for Nanotechnology. American Journal of Nano Research and Applications, 3(1), 2015, pp. 1-5.

[105] Mohamed S. El Naschie: Deriving E $=\mathrm{mc}^{2} / 22$ of Einstein’s Ordinary Quantum Relativity Energy Density from the Lie Symmetry Group SO(10) of Grand Unification of All Fundamental Forces and without Quantum Mechanics. American Journal of Mechanics \& Applications, 2(2), 2014, pp. 6-9.

[106] Mohamed S. El Naschie: Cosserat-Cartan Modification of Einstein-Riemann Relativity and Cosmic Dark Energy Density. American Journal of Modern Physics, 3(2), 2014, pp. 82-87.

[107] Mohamed S. El Naschie: Logarithmic Running of 't Hooft-Polyakov Monopole to Dark Energy. International Journal of High Energy Physics, 1(1), 2014, pp. 1-5.

[108] Mohamed S. El Naschie: Experimentally Based Theoretical Arguments that Unruh’s Temperature, Hawking's Vacuum Fluctuation and Rindler's Wedge Are Physically Real. American Journal of Modern Physics, 2(6), 2013, pp. 357-361.

[109] M. S. El Naschie: The Quantum Gravity Immirzi Parameter-A General Physical and Topological Interpretation. Gravitation and Cosmology, 19(3), 2013, pp. 151-155.

[110] M. S. El Naschie: To Dark Energy Theory from a Cosserat-Like Model of Spacetime. Problems of Nonlinear Analysis in Engineering Systems, 1(41), Vol. 20, 2014, pp. 79-98.

[111] HRH the Prince of Wales, Tony Juniper and Ian Skelly: Harmony: A New Way of Looking at Our World. Harper Collins Publications, London, UK, 2010.

[112] M. S. El Naschie: Casimir-Like Energy as a Double Eigenvalue of Quantumly Entangled System Leading to the Missing Dark Energy Density of the Cosmos. International Journal of High Energy Physics, 1(5), 2014, pp. 55-63.

[113] M. S. El Naschie: On a General Theory for Quantum Gravity. In "Science of The Interface” Editors: H. Diebner, T. Druckry and P. Weibel. Genista Verlag, Tuingen, Germany, 2001, pp. 52-57.

[114] P. Holland: The Quantum Theory of Motion. Cambridge University Press, Cambridge, UK, 1995.

[115] G. 'tHooft: What Is Quantum Mechanics? In "Frontiers of Fundamental Physics” Editors: B. Sidharth and A. Alfonso-Faus. American Institute of Physics, AIP Conference Proceedings No. 905, New York, 2007, pp. 84-102.

[116] M. S. El Naschie: Deterministic Quantum Mechanics versus Classical Mechanical Indeterminism and Nonlinear Dynamics. In "Frontiers of Fundamental Physics” Editors: B. Sidharth and A. Alfonso-Faus. American Institute of Physics, AIP Conference Proceedings No. 905, New York, 2007, pp. 56-63.

[117] L. Susskind and J. Lindesay: An Introduction to Black Holes, Information and the String Theory Revolution. World Scientific, New Jersey, USA, 2005.

[118] R. Penrose: The Road to Reality. Jonathan Cape, London, 2004.

[119] A. Connes: Noncommutative Geometry. Academic Press, San Diego, USA, 1994.

[120] M. S. El Naschie, J.-H. He, S. Nada, L. Marek-Crnjac, M. A. Helal: Golden Mean Computer For High Energy Physics. Fractal Spacetime and Noncommutative Geometry in Quantum and High Energy Physics, 2(2), 2012, pp. 80-93.

[121] M. S. El Naschie, S. Olsen, J. H. He, S. Nada, L. Marek-Crnjac, A. Helal: On the Need for Fractal Logic in High Energy Quantum Physics. International Journal of Modern Nonlinear Theory and Application, 1(3), 2012, pp. 84-92. 